\section{O QUE MEDEM OS RANKINGS UNIVERSITÁRIOS INTERNACIONAIS? Apontamentos teóricos, indicadores e características}

\author{
Samile Andréa de Souza Vanz*
}

RESUMO Os rankings universitários internacionais vem ganhando importância no cenário mundial por simplificar e reduzir grande quantidade de informações acerca de universidades espalhadas por diferentes países. Ao mesmo tempo em que esta constitui sua principal vantagem, reduzir informações advindas de diferentes contextos econômicos, sociais e culturais pode enviesar os resultados. Este artigo apresenta uma revisão de literatura sobre o tema e reúne aspectos importantes para ampliar a discussão acerca dos rankings, debate os indicadores mais utilizados e principais críticas aos rankings universitários league tables. São discutidos os indicadores qualitativos e quantitativos utilizados pelos rankings Shanghai, THE, QS e Leiden. Considerandose a relevância alcançada por tais rankings, conclui-se que é fundamental ampliar a discussão entre a comunidade científica brasileira, a fim de proporcionar maior entendimento sobre o tema.

Palavras-chave: Ciência da Informação. Indicadores. Avaliação universitária. Ranking universitário.
* Doutora em Comunicação e Informação pela Universidade Federal do Rio Grande do Sul, Brasil. Professora do Departamento de Ciências da Informação da Universidade Federal do Rio Grande do Sul, Brasil. Docente permanente do Programa de Pós-graduação em Comunicação e Informação da Universidade Federal do Rio Grande do Sul, Brasil. E-mail: samilevanz@terra.com.br.

\section{INTRODUÇÃO}

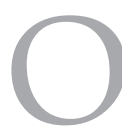

surgimento de rankings universitários globais, ao longo dos últimos anos, colocou os holofotes sobre as universidades, que estão cada vez mais sendo comparadas nacional e internacionalmente. Os rankings certamente ajudaram a promover uma maior responsabilização e melhores práticas de gestão das universidades, que constituem o setor responsável pela maior parte da pesquisa científica brasileira (LETA; GLÄNZEL; THIJS, 2006; PEDROSA, CHAIMOVICH, 2015). A partir do lançamento do Academic Ranking of World Universities (ARWU) em 2003, popularmente conhecido como Ranking de Shanghai, várias outras iniciativas surgiram, como o Leiden Ranking, QS University Rankings, The Times Higher Education (THE) e o U-Multirank.
No Brasil, o Exame Nacional de Cursos ("Provão"), criado em 1996, é considerado o primeiro ranking surgido no cenário nacional e foi um marco na educação superior brasileira, por ter colocado a avaliação na agenda das universidades e da própria sociedade (CALDERÓN; POLTRONIERI; BORGES, 2011). Atualmente, o país dispõe do Ranking Universitário Folha (RUF), publicado anualmente pelo jornal Folha de São Paulo (FRANÇA, 2015); e desde 2014, do Brazilian Research Ranking, que propõe, além da tradicional análise do desempenho das universidades, a mensuração das atividades desenvolvidas nas instituições de pesquisa brasileiras (VOGEL et al., 2014).

Segundo Enserink (2007), os rankings universitários foram inventados pela revista U.S. News \& Words Reports em 1983, com o objetivo de incentivar as matrículas nas universidades mais prestigiadas. No entanto, a primeira 
classificação de universidades é antiga - data de 1870 - feita nos Estados Unidos pela Commission of the US Bureau of Education (RAUGVANGERS, 2011). Algumas metodologias e indicadores comuns atualmente já foram usados há muitos anos, como a análise do número de cientistas eminentes, que fundamentou o ranking de instituições criado por James Catelli em 1910; e o ranking por disciplinas, utilizado pela primeira vez em 1925.

Os rankings principais, denominados league tables, utilizam para suas avaliações os indicadores básicos de produção científica e reputação, coletados respectivamente em bancos de dados internacionais de artigos científicos e através de pesquisas de opinião com acadêmicos e empregadores. Estima-se que os rankings universitários internacionais cobrem entre 1\% e $3 \%$ das universidades, ignorando completamente as demais. Esses percentuais correspondem à cerca de 200 a 500 universidades, dentre um total aproximado de 17.000 universidades do mundo (RAUHVANGERS, 2011; HAZELKORN, 2013).

Em alguns países os rankings têm sido usados para argumentar a favor de um maior investimento no ensino superior (RAUHVANGERS, 2011). Dado o impacto e a importância dos rankings universitários internacionais, é fundamental examinar com detalhes os diferentes tipos existentes, suas características e as fragilidades de cada um deles. Uma análise desta natureza tem um papel importante de orientar um diversificado público - estudantes, professores, pesquisadores, empresas, governos, instituições de ensino interessados em entender como interpretar e processar as informações contidas nos diferentes rankings, bem como identificar as suas limitações. Este artigo apresenta uma revisão de literatura sobre o tema e reúne aspectos importantes para ampliar a discussão acerca dos rankings, como os indicadores mais utilizados e principais críticas aos rankings universitários league tables. O tema, apesar de extensivamente discutido em revistas estrangeiras das áreas de Educação e Cientometria, ainda é incipiente no Brasil. Nas seções que seguem são apresentados alguns apontamentos teóricos sobre os rankings e, em seguida, as características dos rankings que envolvem indicadores qualitativos $\mathrm{e}$ quantitativos. Algumas considerações finais encerram o artigo.

\section{DIFERENTES OLHARES PARA OS RANKINGS UNIVERSITÁRIOS}

A popularização dos rankings estimulou o debate sobre a qualidade e a performance dos sistemas de educação superior, e gerou um grande impacto na sociedade e na internacionalização das universidades (SANZCASADO, 2015). O rápido crescimento e aceitação dos rankings pela sociedade constitui a prova de seu valor. Uma das grandes utilidades dos rankings é servir como instrumento para a escolha de universidades pelos futuros alunos, incluindo os estrangeiros. Os líderes das instituições de ensino superior reconhecem que uma alta posição nos rankings melhora a visibilidade, o que leva ao desenvolvimento de boas estratégias de contratação, produção de conhecimento e marketing (LEITE; PINHO, 2017). Neste sentido, o fornecimento de informação acerca do prestígio das universidades pode resultar na captura de recursos que vão além do financiamento por agências de fomento, como a captura de estudantes, bons pesquisadores e professores. A evolução e aperfeiçoamento dos rankings auxilia os estudantes e professores, que podem decidir, através dos resultados, em que universidade pretendem estudar ou trabalhar, já que a posição ocupada por uma universidade em um ranking pode promover e publicizar as atividades de ensino e educação por ela desenvolvidas (DE FILIPPO et al., 2012).

Safon (2013) aponta que a reputação ou país de origem da universidade são aspectos que exercem influência nos rankings universitários. O autor argumenta que os rankings estão distraindo a atenção de fatores importantes - pesquisa, ensino e terceira missão (empreendedorismo, inovação, transferência de conhecimento e compromisso social) - e colocando a atenção em outros aspectos, obrigando as instituições de pesquisa que desejam melhorar sua posição a alterar seu comportamento para atender necessidades de mercado.

A missão institucional, a qualidade do ensino e o desempenho em pesquisa são alguns itens importantes na avaliação de uma universidade. Uma das grandes vantagens dos rankings universitários é o fato de eles reduzirem diversos fatores em um único indicador objetivo. A popularidade dos rankings deve-se em grande 
parte a sua simplicidade. Paradoxalmente, este é também o principal alvo de críticas (HAZELKORN, 2013). A escolha de quais fatores são importantes e em que proporção devem compor os indicadores é subjetiva e reflete o conceito de qualidade entendido pelos produtores do ranking. Neste sentido, é fundamental conhecer a metodologia e os fatores avaliados por cada ranking.

Segundo Rauhvangers (2011), o fato de os rankings aplicarem os mesmos parâmetros para medir atividades de instituições muito diferentes constitui uma das maiores objeções a eles. Hazelkorn (2013) argumenta que os rankings ignoram o fato de que as universidades são organizações complexas, localizadas em contextos nacionais diversos, baseadas em valores diferentes que atendem às necessidades demográficas, étnicas e culturais de populações variadas. As universidades respondem a um contexto político e econômico em constante mudança e a objetividade dos indicadores quantitativos não contempla as práticas universitárias cotidianas. Os indicadores para avaliação da universidade devem ser legitimados por condições culturais e políticas específicas, quer de cada instituição, quer de um conjunto delas com filiações comuns (MOROSINI et al., 2016).

Hicks et al. (2015, p. 429, tradução nossa) declararam no Manifesto de Leiden: “Em todo o mundo, as universidades estão obcecadas com sua posição nos rankings globais (tais como o Ranking de Shangai e a lista do Times Higher Education), quando estas listas se baseiam em dados que, a nosso juízo, são inexatos e indicadores arbitrários." O Manifesto de Leiden pontua como princípio que "o desempenho deve ser medido de acordo com a missão de investigação da instituição, grupo ou pesquisador". Os autores defendem que não há um modelo de avaliação único que possa ser aplicado a todos os contextos, e que a eleição e uso de indicadores deve considerar os contextos socioeconômicos e culturais e devem estar intimamente relacionados aos objetivos de um programa de pesquisa.

A missão e objetivos da universidade podem, de certa maneira, prever e direcionar a participação de uma instituição em um ranking. Os modelos universitários orientados à internacionalização são os que direcionam as universidades aos rankings internacionais, tendo em vista que são mais voltados à pesquisa e publicação em revistas de alto impacto, à inovação e transferência de conhecimento através de patentes, e à implantação de spin off (DE FILIPPO et al., 2012).

Em um estudo comparativo acerca dos resultados dos rankings de Shanghai e THE, Safon (2013) afirma a existência de um fator subjacente aos indicadores utilizados, visto que mais de $73 \%$ da variância dos resultados apresentados por estes dois rankings pode ser explicada por seis fatores: universidades com alta reputação, localizadas nos Estados Unidos ou em países que falam inglês, com forte dedicação à pesquisa, que tem a sua disposição orçamentos abundantes e orientadas às ciências duras.

Hazelkong (2013), preocupada com o direcionamento que os rankings podem estar dando aos esforços das universidades, argumenta que pode acontecer uma priorização desenfreada de atividades que resultem positivamente em subida de posições. Seus resultados de pesquisa mostram que os rankings estão influenciando líderes: $76 \%$ dos gestores universitários reconhecem valer-se dos rankings para monitorar o que outras instituições de seu país estão fazendo e $50 \%$ afirmam monitorar instituições internacionais. Ainda, 40\% reconhece utilizar os rankings para avaliar a validade de convênios de cooperação.

Mais recentemente, Elken, Howdhaugen e Stensaker (2016) em um estudo dos documentos e planos estratégicos, além de entrevistas com os gestores das universidades nórdicas, encontraram resultados que mostram que os rankings têm um impacto modesto nos gestores e nas ações estratégicas das universidades nórdicas estudadas, com poucos sinais de alterações da identidade dessas universidades. Segundo os autores, a prática estabelecida por vários rankings internacionais de converter dados brutos em pontuações ponderadas em relação a fatores complexos, como por exemplo, em relação ao total de pontos obtido pela universidade com melhor desempenho em determinado indicador, pode comprometer a possibilidade dos rankings servirem como instrumento de benchmarking. O entendimento das pontuações não é nada intuitivo e sem o uso de dados brutos não é possível melhorar a qualidade das universidades (ELKEN; HOWDHAUGEN; STENSAKER, 
2016). O Leiden Ranking é um dos poucos que fornece informações fáceis de entender, baseadas em dados brutos e que possibilitam muitas comparações de performance entre diferentes universidades. Seu entendimento intuitivo permite que os dados sejam usados para orientar decisões e ações das universidades (ELKEN et al., 2016).

Apesar dos preconceitos evidentes, fragilidades e falhas, os rankings estão instituídos socialmente e desfrutam de um alto nível de aceitação social devido à simplicidade de sua informação. Para as universidades, os rankings são úteis para comparar o desempenho em âmbito nacional e internacional, reorganizar a estrutura e procedimentos de pesquisa e direcioná-las para áreas mais vantajosas, modernizá-las e facilitar a transparência e prestação de contas.

\begin{abstract}
O sucesso dos rankings acontece devido à globalização da educação superior onde a universidade pode competir internacionalmente por recursos econômicos e humanos. As instituições de educação superior estão utilizando estes rankings como ferramenta de autopromoção, mostrando sua excelência em educação, pesquisa ou negócios. O número de visitantes aos sites dos rankings é da ordem de milhões por ano e os estudantes os utilizam como guias para escolher as universidades onde vão se candidatar (especialmente as estrangeiras). Esta publicidade também é dirigida para aumentar $\circ$ potencial para receber financiamento e empregar pesquisadores de alta qualidade que permitem elevar a posição da universidade no mercado educacional. Do ponto de vista científico, as universidades precisam dos rankings para aumentar sua performance científica participando de projetos de pesquisa internacionais e atraindo estudantes e pesquisadores. (AGUILLO et al., 210, p. 244)
\end{abstract}

De acordo com Rauhvagers (2011), há vários tipos de rankings universitários: aqueles cujo propósito principal é produzir league tables das melhores universidades, como o Ranking de Shanghai e THE; aqueles cujo foco é a performance em pesquisa, como o Leiden Ranking; aqueles que utilizam vários indicadores sem o objetivo de produzir um score, como o alemão CHE; e por fim, aqueles que medem a visibilidade na web, como o Webometrics. Os rankings nacionais desempenham um papel importante, pois comparam instituições dentro de um mesmo contexto, permitindo uma confrontação interessante para o adequado estabelecimento de estratégias para desenvolvimento das universidades. Além disso, permitem uma avaliação mais profunda que não é alcançada pelos rankings internacionais.

Conforme comentado, os rankings fundamentamse em indicadores quantitativos ou qualitativos e, em alguns casos, utilizam ambos indicadores. A próxima seção discute características específicas dos rankings que envolvem indicadores qualitativos - as avaliações de opinião. Na seção seguinte, apresentam-se aspectos pertinentes aos rankings baseados unicamente em indicadores quantitativos.

\section{RANKINGS QUE ENVOLVEM AVALIAÇÃO DE OPINIÃO}

Os rankings que envolvem avaliações de opinião são duramente criticados pela literatura por sua subjetividade, como é o caso do THE e do QS University Rankings. Conforme Rauhvagers (2011), as surveys não medem nada além da reputação das universidades e apenas cerca de 5\% dos acadêmicos questionados realmente respondem às pesquisas, reduzindo muito a representatividade das mesmas. O autor ainda argumenta que a prática de alguns rankings, de solicitar ao entrevistado que aponte universidades de prestígio em listas prontas oferecidas nos questionários, induz o entrevistado, tendo em vista que muitas universidades e até mesmo países inteiros são omitidos. O "prestígio histórico" desencadeia o chamado "efeito persistência", já que a reputação de uma universidade depende, em parte, de suas classificações passadas (FERNANDES; NUNES, 2011).

Andrade (2011) concorda com a subjetividade dos rankings que envolvem opiniões de especialistas, por considerar pouco provável que alguém conheça e seja capaz de avaliar adequadamente os diferentes aspectos das universidades com diferentes perfis e espalhadas pelo mundo. O autor ainda aponta que a tentativa usada para resolver 
este problema, qual seja, ampliar ao máximo o número de respondentes, acaba por criar outra dificuldade: compatibilizar num único ranking as respostas dadas por diferentes indivíduos sobre as diferentes instituições. Isto porque eles não tem o mesmo grau de exigência, nem avaliam da mesma maneira e nem atribuem o mesmo valor para as distintas características das instituições.

Outra importante crítica feita aos rankings que utilizam indicadores de reputação são os vieses regionais, já que a maioria dos questionários devolvidos provém de países que falam inglês (SAFON, 2013). Quando se trata dos questionários enviados às próprias universidades, Enserink (2007), comenta que muitas delas não declaram seus dados aos rankings no intuito de boicotá-los, atuando junto à um movimento de oposição aos rankings que reúne universidades de renome internacional.

No Ranking THE, o cálculo do indicador reputação acadêmica baseia-se na Academic Reputation Survey que reúne respostas de mais de uma centena de países (THE..., 2016). Em casos de países sub-representados nas respostas do survey, a equipe do THE procede à uma ponderação do conjunto de respostas para refletir mais de perto a distribuição geográfica real dos pesquisadores, com base nos dados das Nações Unidas.

$\mathrm{Na}$ survey do Ranking THE, os pesquisadores são questionados acerca das áreas de sua especialidade. O survey convida o pesquisador, com base em sua própria experiência, a nomear até 15 universidades que considera top nas categorias pesquisa e ensino (THE, 2016). Uma das grandes críticas ocorre pelo fato das listas serem pré-selecionadas pelo próprio Ranking THE, e por isso, muitas universidades e países inteiros ficam totalmente excluídas (RAUHVARGERS, 2011). O Ranking THE contrapõe esta crítica informando que as listas são montadas com base no sucesso obtido pelas universidades em rankings domésticos, nas sugestões feitas pelos empregadores entrevistados, em um balanço geográfico e admissão direta das instituições que solicitam a avaliação do Ranking. Desde 2008 os acadêmicos e empregadores respondem acerca das instituições mundiais e de seu país em separado para evitar parcialidade.

Segundo Piro e Sivertsen (2016), existem dois fatores que explicam a variação em posições no Ranking THE: o indicador de citações e o número de indicações na pesquisa de reputação. Os dados da pesquisa não são públicos e por isso, não há como conhecer estes resultados. O próprio THE (2016) argumenta que só publica os resultados das 50 melhores universidades pois a partir desta posição as diferenças são pequenas entre as universidades. Piro e Sivertsen (2016) contrapõe, afirmando que há outras duas razões para que os resultados não sejam públicos. Primeiro, os resultados evidenciarão a dependência do THE em um único indicador que pontua desproporcionalmente mais baixo a maior parte das universidades mundiais. Segundo, e também relacionado ao baixo número de indicações obtidas pela maior parte das universidades, os resultados são passíveis de muita flutuação anual, tendo em vista que alguns votos a mais ou a menos causam um grande impacto no score da universidade. Bookstein et al. (2010) reforçam as críticas quanto a instabilidade dos indicadores de reputação e aluno-to-staff utilizados pelo THE. Os autores argumentam que cerca de $95 \%$ das universidades se mantém, ano após ano, entre as 200 primeiras no ranking, porém, a grande flutuação entre as posições não é aceitável.

\section{RANKINGS BASEADOS EM DADOS QUANTITATIVOS}

Ao enfatizar a produção científica, os rankings criam maneiras de comparar o desempenho de universidades globais, tendo em vista que produção científica e citação são indicadores universais baseados em dados sólidos e repletos de significado que podem minimizar as diferenças nos contextos educacionais e culturais (ENSERINK, 2007). Segundo o autor, rankings como o de Shanghai e Leiden utilizam dados públicos e objetivos como estratégia para evitar a possível manipulação dos dados enviados pela própria universidade e a coleta imprecisa de informações nas pesquisas de opinião com especialistas e recrutadores.

No entanto, rankings baseados em indicadores objetivos também têm suas desvantagens. A escolha de indicadores baseados exclusivamente na produção científica coloca todas as outras atividades da universidade em posição de menor importância ou totalmente a 
parte. Essa é uma discussão importante tendo em vista que o sistema educacional superior fundamenta-se na tríade ensino-pesquisaextensão. Nas universidades brasileiras, vigora o modelo de trabalho baseado nestas três atividades e por isso, avaliar exclusivamente a atividade de pesquisa pode prejudicar toda a comunidade universitária que atua em atividades que vão muito além.

$\mathrm{Na}$ opinião de Safon (2013), rankings que consideram o número absoluto de artigos publicados, como o Ranking de Shangai, beneficiam as universidades maiores. Esta característica do Ranking de Shanghai pode distorcer as discussões acerca da excelência universitária e levar gestores a promover fusões entre universidades, com o objetivo de ampliar o tamanho das mesmas.

Como ponderam Docampo e Cram (2015), a noção de "universidade de pesquisa" (research universities), conforme estabelecidas por Rawlings et al. (2013) no Hefei Statement, considera características como qualidade, amplitude e profundidade, as quais reforçam a ideia de abrangência e tamanho. As research universities são responsáveis por uma proporção substancial do esforço de pesquisa de uma nação, no entanto, compõem somente uma parcela pequena de faculdades e de universidades. A ideia que se extrai deste documento é, portanto, que a orientação para a pesquisa não é obrigatória para todas as universidades.

Van Raan (2005) acrescenta mais um argumento - que o uso de indicadores bibliométricos pelos rankings de instituições de pesquisa é inadequado por estes apresentarem inúmeros problemas cruciais na avaliação de performance em pesquisa. Decorridos mais de dez anos desde a publicação do artigo onde o autor defende um ponto de vista contrário aos rankings, especificamente ao Ranking de Shangai, a grande maioria dos argumentos de Van Raan (2005) continua válida. Os indicadores bibliométricos não estão suficientemente desenvolvidos para que possam servir aos rankings, porém, o principal problema, na opinião do autor, advém do fato de eles serem usados por pessoas que não tem competência e experiência nos estudos quantitativos da ciência.

Van Raan (2005) aponta problemas técnicos que prejudicam uma avaliação generalizada das instituições de pesquisa. Um deles diz respeito a dificuldade em relacionar documentos citantes e citados. Segundo o autor, até 30\% das citações se perdem por inconsistência em nomes de autores, periódicos e datas. A variação dos nomes das instituições e entradas por nomes de departamentos também prejudica a identificação das citações, além da dificuldade na vinculação dos hospitais universitários.

$\mathrm{O}$ uso dos indicadores de produção científica gera um irrefutável preconceito por favorecer universidades fortes em pesquisa nas áreas das Ciências Naturais e Medicina, subrepresentar as universidades de Engenharia e Ciências Sociais, e ignorar, quase ou completamente, as Humanidades. Nesse sentido, comparar os resultados de uma determinada instituição com outra é problemático (BORNMANN; MOYA-ANEGÓN， 2014; HAZELKORN, 2013).

A cobertura das bases de dados utilizadas também é apontada, por Van Raan (2005), como um problema, tendo em vista que as Engenharias, Ciências Sociais e Humanidades são áreas pouco indexadas, o que altera o desempenho de universidades com perfil dedicado a estas áreas. $\mathrm{O}$ uso de bases de artigos internacionais privilegia aquelas universidades especializadas nas ciências duras ou biomédicas, em detrimento das universidades generalistas que atuam em várias áreas do conhecimento. No intuito de minimizar este problema o Ranking de Shanghai pontua duplamente o impacto de cada publicação indexada no Social Science Citation Index. De qualquer forma, há áreas onde a publicação em revistas internacionais não faz sentido, como o Direito, o que coloca em desvantagem as universidades que incluem estas disciplinas e cuja língua nativa não é o inglês (SAFON, 2013).

O idioma, apesar de não constituir um indicador oficial dos rankings universitários, é apontado pela literatura com um aspecto subjacente de grande influência. Resultados de pesquisa publicados em outras línguas que não o inglês são menos lidos e menos citados. Além disso, periódicos que publicam artigos em outras línguas que não o inglês também perdem em visibilidade, internacionalidade, impacto e prestígio, e possuem uma baixa representatividade em bases de dados internacionais utilizadas para os cálculos dos rankings (SAFON, 2013). 
O peso implícito da idade das universidades volta a discussão quando se analisam os indicadores relacionados a concessão de prêmios Nobel e Medalhas Field utilizados pelo Ranking de Shanghai (DOBROTA; DOBROTA, 2016). A credibilidade do Ranking é questionada pela crítica que se sustenta no longo período abrangido por estes indicadores: por serem considerados prêmios concedidos até 100 anos atrás, as universidades mais antigas são privilegiadas (SAFON, 2013).

Outro indicador quantitativo utilizado pelo Ranking de Shanghai é o número de pesquisadores mais citados proveniente da lista Highly Cited Researchers. Bornmann e Bauer (2015) defendem o uso deste indicador, pois argumentam que o trabalho científico e a atribuição de sucesso acontece em nível de indivíduos. Na opinião dos autores, contar o número de pessoas de sucesso de uma instituição é mais razoável do que contar o número de publicações mais citadas. No entanto, algumas universidades mantém vários pesquisadores presentes na lista Highly Cited Researchers através de contratos parciais, como por exemplo, aqueles realizados por universidades na Arábia Saudita. A King Abdulaziz University é um dos casos apontados por Bornmann e Bauer (2015), pois aparece muito bem posicionada na lista Highly Cited Researchers e no entanto, quando a contagem é feita de forma fracionada, a posição cai, tendo em vista que são pesquisadores que tem mais de uma filiação. Apesar de utilizar uma metodologia para sanar este problema o Ranking de Shanghai não elimina por completo estas discrepâncias.

O tamanho da universidade é apontado como um aspecto subjacente importante para o Ranking de Shanghai (PIRO; SIVERTSEN, 2016). Docampo e Cram (2015) relatam que em torno de 30\% da variação do Ranking de Shanghai é atribuída ao tamanho da universidade e que o indicador utilizado para compensar o tamanho não provoca muita diferença no score final, que tem um peso de apenas $10 \%$ no score total. Segundo Rauhvangers (2011), isso significa que o Ranking reflete a força das universidades, porém as menores, mesmo com alto nível de excelência, tem uma chance muito pequena de aparecer. Este indicador é considerado o indicador mais frágil do Ranking de Shanghai, pois o entendimento do que é um acadêmico em tempo integral varia de instituição para instituição e pode incluir, por exemplo, professores temporários (substitutos), visitantes ou pós doutorandos (FLORIAN, 2007).

Para contrapor esta ideia, há um argumento de que os indicadores dependentes de tamanho não ocultam a excelência das instituições. Poucas universidades que estão entre as maiores do mundo estão listadas entre as primeiras 500 do Ranking de Shanghai, e muitas que estão ranqueadas nas melhores posições não estão sequer entre as maiores do país onde estão localizadas. Nesse sentido, Docampo e Cram (2015) argumentam que além do tamanho, outro fator direciona o Ranking de Shanghai, denominado pelos autores como "qualidade", e entendido como a capacidade de atrair e reter eminentes pesquisadores internacionais.

Segundo Safon (2013), o Ranking THE leva em consideração a medida de produtividade ao invés do número total de artigos, o que, de certa maneira, corrige o problema do tamanho da universidade, pois pondera o volume de artigos pelo volume de professores. No entanto, o Ranking embasa cerca de um terço de sua avaliação na reputação proveniente de avaliações de opinião, o que por sua vez é um indicador relacionado ao tamanho da instituição (RAUHVARGERS, 2011).

Manganote, Schulz e Cruz (2016) compartilham esta ideia e argumentam que é necessário considerar a forma como a pesquisa é desenvolvida para avaliar se o indicador citação pode ser usado para definir rankings individuais ou institucionais. Os resultados apresentados pelos autores comprovaram o efeito positivo dos artigos da área de Física de Altas Energias nos resultados de output e citação, tanto em nível institucional quanto de país. Neste sentido, a participação em grandes colaborações (big science) pode distorcer resultados de universidades com pequeno volume de output. Segundo os autores, a Física de Altas Energias influencia positivamente o desempenho das universidades nos Ranking THE, apesar da metodologia divulgada pelo ranking afirmar o contrário.

\section{CONSIDERAÇÕES FINAIS}

Nas últimas décadas, as universidades tornaram-se maiores, mais populares e mais globais, o que ajuda a explicar o interesse 
e a influência dos rankings na atualidade. Estabelecer comparações entre universidades não é um exercício simples. É necessário considerar variáveis como tamanho, história e contexto de criação, missão e objetivos. Neste sentido, não basta olhar o resultado de um determinado ranking, é fundamental entender que tipo de informação cada um fornece e com base em quais indicadores seu resultado esta baseado.

A avaliação das universidades é parcial quando se fundamenta em indicadores universais ou uma composição deles, o que acontece inevitavelmente com todos os rankings. Como consequência, a ordem de um ranking pode variar em função do produto escolhido. Isto porque uma instituição pode naturalmente ser melhor em um indicador em detrimento a outros, ou focar as suas ações para alcançar um melhor desempenho num indicador específico de forma a ser melhor classificada no ranking. $O$ fato é que não existe um consenso sobre um único indicador.

O prestígio de uma universidade é construído ao longo de vários anos e nesse aspecto, muitas universidades brasileiras ainda trabalham na construção de sua imagem, pois são muito novas quando comparadas às universidades internacionais bem posicionadas nos rankings. Além disso, há vantagens inerentes ao prestígio, como a percepção de qualidade por parte dos alunos, satisfação por parte dos professores e sentimento de orgulho de pertencer à instituição. A atração de financiamento também pode ser apontada como uma consequência.

É importante que as universidades tenham clareza dos seus objetivos e saibam estabelecer prioridades. Enquanto algumas instituições podem competir no cenário global, outras estão inseridas claramente em um contexto nacional. Neste caso, não há razão para lamentar a ausência ou a queda em posições nos rankings universitários globais se o objetivo principal da universidade não é a pesquisa e produção científica, que constituem um indicador de grande peso na maioria dos rankings internacionais. A avaliação da universidade deve ser concebida em relação aos objetivos da instituição.

As universidades brasileiras são recentes quando comparadas as universidades europeias, e publicam um grande volume de resultados de pesquisa em periódicos nacionais em português. Grande parte delas possui uma característica peculiar - a gratuidade - o que basta para atrair alunos brasileiros. Mesmo neste contexto, não se pode ignorar os rankings universitários e o impacto que eles vêm causando na avaliação dos sistemas de ensino superior e nas políticas de C\&T. Os rankings atraem atenção midiática, justificando que as universidades os estudem criticamente. Eles podem ser ferramentas úteis por reunirem informação quantitativa, basta que tenhamos discernimento para escolher rankings que melhor se adaptem ao perfil da universidade que desejamos avaliar. Por isso é fundamental ampliar a discussão sobre o tema entre a comunidade brasileira.

Artigo recebido em 23/02/20I8 e aceito para publicação em I2/03/20।8

\section{WHAT DO THE INTERNATIONAL UNIVERSITY RANKINGS MEASURE? theoretical notes, indicators and characteristics}

ABSTRACT The international university rankings have been gaining importance in the world stage by simplifying and reducing a large amount of information concerning universities spread in different countries. While that constitutes its main advantage, reducing information coming from different economic, social and cultural contexts may mislead the results. This paper presents a literature review on the subject and gathers important aspects to expand the rankings discussion, it debates the indicators used more frequently and the main critics to the league tables university rankings. It discusses the qualitative and quantitative indicators used by Shanghai, $T H E, Q S$ and Leiden rankings. Considering the relevance obtained by such rankings, it concludes that is fundamental to expand the discussion in order to provide a large understanding about the topic.

KEYWORDS: Information Science. Indicators. University evaluation. University Ranking. 


\section{REFERÊNCIAS}

AGUILLO, Isidro F.; BAR-ILLAN, Judit; LEVENE, Mark; ORTEGA, José Luis. Comparing university rankings. Scientometrics, Amsterdam, v. 85, p. 243-256, 2010.

ANDRADE, E.C. Rankings em Educação: tipos, problemas, informações e mudanças: análise dos principais rankings oficiais brasileiros. Estudos Econômicos, São Paulo, v. 41, n. 2, p. 323-343, jun. 2011.

BOOKSTEIN, Fred L. et al. Too much noise in the Times Higher Education rankings. Scientometrics, Amsterdam, v. 85, p. 295-299, 2010 .

BORNMANN, Lutz.; MOYA-ANEGÓN, F. What proportion of excellent papers makes an institution one of the best worldwide? Specifying thresholds for the interpretation of the results of the SCImago Institutions rankings and the Leiden Ranking. Journal of the Association for Information Science and Technology, v. 65, n. 4, p. 732-736, 2014.

BORNMANN, Lutz; BAUER, Johann. Which of the world's institutions employ the most highly cited researchers? An analysis of the data from highlycited.com. Journal of the Association for Information Science and Technology, v. 66, n. 10, p. 2146-2148, 2015.

CALDERÓN, A.I.; POLTRONIERI, H.; BORGES, R.M. Os rankings na educação superior brasileira: políticas de governo ou de Estado? Ensaio: aval. Pol. Publ Educ, Rio de Janeiro, v. 19, n. 73, p. 813826, out./dez. 2011.

CENTRE FOR SCIENCE AND TECHNOLOGY STUDIES. Leiden Ranking. Disponível em: $<$ www.leidenranking.com>. Acesso em: 01 jun. 2016.

DE FILIPPO, Daniela et al. Visibility in international rankings. Strategies for enhancing the competitiveness of Spanish universities. Scientometrics, Amsterdam, v.93, p 949-966, 2012.
DOBROTA, M.; DOBROTA, M. ARWU Ranking uncertainty and sensitivity: what if the Award Factor was excluded? Journal of the Association for Information Science and Technology, v. 67, n. 2, p. 480-482, 2016

DOCAMPO, Domingo. Reproducibility of the Shanghai academic ranking of world universities results. Scientometrics, Amsterdam, v. 94, n. 2, p. 567-587, 2013.

DOCAMPO, Domingo; CRAM, Lawrence. On the effects of institutional size in university classifications: the case of the Shanghai ranking. Scientometrics, Amsterdam, v. 102, p. 1325-1346, 2015.

ENSERINK, Martin. Who Ranks the University Rankers? Science, v. 317, n. 5841, 24 Ago. 2007, p. 1026-1028. [doi: 10.1126/science.317.5841.1026]

ELKEN, Mari; HOVDHAUGEN, Elisabeth; STENSAKER, Bjorn. Global rankings in the Nordic region: challenging the identity of research-intensive universities? Higher Education, Washington, Jan. 2016. Disponível em: < http://link.springer.com/article/10.1007/ s10734-015-9975-6>. Acesso em: 02 jan. 2017.

FERNANDES, Ivanildo; NUNES, Edson. Rankings Internacionais: a irresistível polêmica em torno de seus sentidos e metodologias. Documento de trabalho n. 97. Rio de Janeiro: Observatório Universitário, 2011.

FLORIAN, Razvan V. Irreproducibility of the results of the Shanghai academic ranking of world universities. Scientometrics, Amsterdam, v. 27, n. 1, p. 25-32, 2007.

FRANÇA, Carlos Marshal. Rankings universitários promovidos por jornais no espaço ibero-americano: El Mundo (Espanha), El Mercurio (Chile) e Folha de São Paulo (Brasil). 2015. 225 p. Dissertação (Mestrado em Educação). Pontifícia Universidade Católica de Campinas. Programa de Pós-Graduação Stricto Sensu em Educação. Campinas. 2015.

HAZELKORN, Ellen. How Rankings are Reshaping Higher Education. In: CLIMENT, 
V.; MICHAVILA, F.; RIPOLLÉS , M. (Eds.). Los rankings universitarios, Mitos y Realidades. Madrid: Técnos, 2013.

HICKS, Diana et al. The Leiden Manifesto for research metrics. Nature, v. 520, p. 429-431, 2015.

LEITE, Denise; PINHO, Isabel. Evaluating collaboration networks in higher education research: drivers of excellence. Berlin Heidelberg : Springer, 2017. 129 p.

LETA, Jacqueline.; GLÄNZEL, Wolfgang.; THIJS, Bart. Science in Brazil. Part 2: sectoral and institutional research profiles. Scientometrics, Amsterdam, v. 67, n.1, p. 87-105, 2006.

MANGANOTE, Edmilson J.T.; SCHULZ, Peter A.; CRUZ, Carlos Henrique de Brito. Effect of high energy physics large collaborations on higher education institutions citations and rankings. Scientometrics, v. 109, p. 813-826, 2016.

MOROSINI, Marília Costa et al. A qualidade da educação superior e o complexo exercício de propor indicadores. Revista Brasileira de Educação, v. 21, n. 64, p. 13-37, jan./mar. 2016.

PEDROSA, Renato Hyuda de Luna; Chaimovich, Hernan. Brazil. In: Relatório de ciência da Unesco: rumo a 2030: visão geral e cenário brasileiro. Paris: Unesco Publishing, 2015, 58 p. Disponível em: <http://unesdoc.unesco.org/ images/0023/002354/235407por.pdf > . Acesso em: 17 set. 2017.

PIRO, Fredrik Niclas; SIVERTSEN, Gunnar. How can differences in international university rankings be explained? Scientometrics, Amsterdam, v. 109, p. 2263-2278, 2016.

RAUHVARGERS, Andrejs. Global university rankings and their impact. European University Association, 2011. 85 p.

RAWLINGS, Hunter, et al. The ten characteristics of contemporary research Universities. 2013. Disponível em: < http:// www.leru.org/files/news/Hefei_statement. pdf>. Acesso em: 16 jan. 2017.
SAFON, Vicente. What do global university rankings really measure? The search for the $X$ factor and $X$ entity. Scientometrics, Amsterdam, v. 97, p. 223-244, 2013.

SANZ-CASADO, Elias. et al. Rankings nacionales elaborados a partir de múltiples indicadores frente a los de índices sintéticos. Revista Española de Documentación Científica, Madrid, v. 36, n.3, 2013.

SANZ-CASADO, Elias. (coord.). Guía de buenas prácticas para la participación de las universidades españolas en los rankings internacionales. Madrid: Ministerio de Educación, Cultura y Deporte. 2015. 101 p.

THE TIMES HIGHER EDUCATION RANKING. World University Rankings 2016-2017 Methodology. 2016. Disponível em: <https:// www.timeshighereducation.com/worlduniversity-rankings/methodology-worlduniversity-rankings-2016-2017>. Acesso em: 23 nov. 2016.

VAN RAAN, Anthony F. J. Fatal attraction: conceptual and methodological problems in the ranking of universities by bibliometric methods. Scientometrics, Amsterdam, v. 62, n. 1, p. 133143, 2005.

VOGEL, Michely Jabala Mamede. Como ler um ranking: a proposta do Brazilian Research Ranking. 2014. In: Anais ... Encontro Nacional de Pesquisa em Ciência da Informação. Belo Horizonte, 2014. Disponível em: < http:// enancib2014.eci.ufmg.br/documentos/anais/ anais-gt7 >. Acesso em: 25 jan. 2017.

\section{Financiamento}

Este trabalho recebeu financiamento do Conselho Nacional para Desenvolvimento Científico e Tecnológico (CNPq). 